\title{
Kähler manifolds with quasi-constant holomorphic curvature
}

\section{Włodzimierz Jelonek}

Published online: 27 March 2009

(C) Springer Science+Business Media B.V. 2009

\section{Erratum to: Ann Glob Anal Geom DOI: 10.1007/s10455-009-9154-z}

The formula (9.4) in the paper is not correct. Therefore, the formula for the curvature in Theorem 9.1 should be

$$
\begin{aligned}
R( & \left.X^{*}+\alpha H+\beta J H, J X^{*}+\alpha J H-\beta H, J X^{*}+\alpha J H-\beta H, X^{*}+\alpha H+\beta J H\right) \\
= & a^{4} R(H, J H, J H, H)-a^{2} \beta^{2} R(H, J H, H, J H)+\beta^{4} R(J H, H, H, J H) \\
- & \alpha^{2} \beta^{2} R(J H, H, J H, H)+\alpha^{2} R\left(H, J X^{*}, J H, X^{*}\right)+\alpha^{2} R\left(H, J X^{*}, J X^{*}, H\right) \\
+ & \alpha^{2} R\left(X^{*}, J H, J X^{*}, H\right)+\alpha^{2} R\left(X^{*}, J H, J H, X^{*}\right)+\beta^{2} R\left(J H, J X^{*},-H, X^{*}\right) \\
& \beta^{2} R\left(J H, J X^{*}, J X^{*}, J H\right)+\beta^{2} R\left(X^{*}, H, H, X^{*}\right)+\beta^{2} R\left(X^{*},-H, J X^{*}, J H\right) \\
& +2 \alpha^{2} R\left(H, J H, J X^{*}, X^{*}\right)-2 \beta^{2} R\left(J H, H, J X^{*}, X^{*}\right)+R\left(X^{*}, J X^{*}, J X^{*}, X^{*}\right) \\
= & \left|X_{\mathcal{D}}\right|^{4} R(H, J H, J H, H)+8\left|X_{\mathcal{D}}\right|^{2} R\left(H, J X^{*}, J X^{*}, H\right)+R\left(X^{*}, J X^{*}, J X^{*}, X^{*}\right) \\
= & \left|X_{\mathcal{D}}\right|^{4} R(H, J H, J H, H)+8\left|X_{\mathcal{D}}\right|^{2}\left(1-\left|X_{\mathcal{D}}\right|^{2}\right) R\left(J H, \tilde{X}^{*}, \tilde{X}^{*}, J H\right) \\
& +\left(1-\left|X_{\mathcal{D}}\right|^{2}\right)^{2} R\left(\tilde{X}^{*}, J \tilde{X}^{*}, J \tilde{X}^{*}, \tilde{X}^{*}\right)
\end{aligned}
$$

Everything else in the paper remains correct.

The online version of the original article can be found under doi:10.1007/s10455-009-9154-z.

W. Jelonek $(\bowtie)$

Institute of Mathematics, Cracow University of Technology, Warszawska 24, 31-155 Kraków, Poland e-mail: wjelon@pk.edu.pl 